PROCEEDINGS OF THE

AMERICAN MATHEMATICAL SOCIETY

Volume 134, Number 3, Pages 645-652

S 0002-9939(05)08175-X

Article electronically published on August 29, 2005

\title{
WITT KERNELS OF BILINEAR FORMS FOR ALGEBRAIC EXTENSIONS IN CHARACTERISTIC 2
}

\author{
DETLEV W. HOFFMANN
}

(Communicated by Bernd Ulrich)

In memory of Professor Martin Kneser

\begin{abstract}
Let $F$ be a field of characteristic 2 and let $K / F$ be a purely inseparable extension of exponent 1 . We determine the kernel $W(K / F)$ of the natural restriction map $W F \rightarrow W K$ between the Witt rings of bilinear forms of $F$ and $K$, respectively. This complements a result by Laghribi who computed the kernel for the Witt groups of quadratic forms for such an extension $K / F$. Based on this result, we will determine $W(K / F)$ for a wide class of finite extensions which are not necessarily purely inseparable.
\end{abstract}

\section{INTRODUCTION}

Throughout this note, we will only consider fields of characteristic 2 . Let $K$ be a finite purely inseparable multiquadratic extension of a field $F$, in other words, $K=F\left(\sqrt{a_{1}}, \cdots, \sqrt{a_{n}}\right)$. In [7, Laghribi computed the kernel $W_{q}(K / F)$ of the natural map (induced by scalar extension) $W_{q} F \rightarrow W_{q} K$ between the Witt groups of nonsingular quadratic forms over $F$ and $K$, respectively. He shows that

$$
W_{q}(K / F)=\sum_{i=1}^{n}\left\langle 1, a_{i}\right\rangle \otimes W_{q} F,
$$

where $\left\langle 1, a_{i}\right\rangle$ is understood to be a binary bilinear form in its diagonal notation, and $W_{q} F$ is considered as a $W F$-module. The proof, although elementary as such, uses as nontrivial ingredients the analogues in characteristic 2 of the Arason-Pfister Hauptsatz due to Baeza [1, Satz 4.1], and of the Milnor conjecture due to Kato [6]. For multiquadratic extensions that are not purely inseparable, results seem to be much harder to obtain, and the precise kernels are only known in the quadratic and biquadratic case. For more details, we refer the reader to Laghribi's article and the references there.

Here, we will determine the kernel $W(K / F)$ of such a purely inseparable multiquadratic extension $K / F$ for the restriction map $W F \rightarrow W K$ between the Witt rings of bilinear forms. It turns out that the behaviour of bilinear forms under such

Received by the editors October 10, 2004.

2000 Mathematics Subject Classification. Primary 11E04; Secondary 11E81, 12F15.

Key words and phrases. Quadratic form, bilinear form, Pfister form, Witt ring, excellent extension, purely inseparable extension, exponent of an inseparable extension, balanced extension.

The research on this paper was supported in part by the European research network HPRNCT-2002-00287 "Algebraic K-Theory, Linear Algebraic Groups and Related Structures".

(C)2005 American Mathematical Society Reverts to public domain 28 years from publication 
extensions can be studied in a truly elementary way, without using any deep results which become necessary when dealing with quadratic forms.

Our main result is the following:

Theorem 1.1. Let $K / F$ be a field extension such that $K^{2} \subset F$. Then $W(K / F)$ is the ideal in $W F$ generated by $\left\{\langle 1, t\rangle \mid t \in K^{* 2}\right\}$.

Note that a field extension $K / F$ with $K^{2^{n}} \subset F$ is said to have exponent $\leq n$. So our result is a statement concerning field extensions of exponent 1 . Such a field extension is obviously algebraic and purely inseparable. It turns out that the inseparable extensions $K / F$ we consider have the property that they are excellent, i.e., if $\varphi$ is a bilinear form over $F$, then there exists a bilinear form $\psi$ over $F$ such that the anisotropic part of $\varphi_{K}$ is isometric to $\psi_{K}$. The above theorem and the excellence will be shown in Section 3 .

In the case of a finite purely inseparable multiquadratic extension $K / F$, we will give a description of $W(K / F)$ in terms of the annihilator ideal of a certain bilinear Pfister form naturally attached to such an extension (Section 4), and we will generalize this result in Section 5 to an even wider class of finite extensions which are not necessarily purely inseparable and which may contain purely inseparable subextensions of exponent greater than one. It turns out that all extensions in this class are excellent in the above sense, but we will also give an example of an extension of degree 6 that is not excellent.

\section{Terminology And Definitions}

Before turning to the proofs, let us fix some terminology and recall some basic facts about bilinear forms as can be found, for example, in Chapters 1 and 2 of Pfister's book [8]. By a form we will always mean a finite-dimensional regular symmetric bilinear form.

$\alpha \cong \beta$ denotes isometry of the two forms $\alpha$ and $\beta$. The orthogonal sum (resp. the product) of $\alpha$ and $\beta$ is written $\alpha \perp \beta$ (resp. $\alpha \otimes \beta$ ).

A form $\beta$ is said to be isotropic if it represents 0 nontrivially, anisotropic otherwise, and $D_{F}(\beta)=\{\beta(x, x) \mid x \in V \backslash\{0\}\}$ denotes the set of all values represented nontrivially by $\beta$ (here, $V$ denotes the underlying $F$-vector space). Thus, $\beta$ being isotropic is equivalent to $0 \in D_{F}(\beta)$. We put $D_{F}(\beta)^{*}=D_{F}(\beta) \cap F^{*}$ and $D_{F}(\beta)_{0}=D_{F}(\beta) \cup\{0\}$.

A 2-dimensional isotropic form is called a metabolic plane, in which case one can always find a basis such that the Gram matrix with respect to that basis is of the shape

$$
\left(\begin{array}{ll}
a & 1 \\
1 & 0
\end{array}\right)
$$

for some $a \in F$. If $a=0$, this is called a hyperbolic plane and denoted by $\mathbb{H}$. A form $\beta$ is said to be metabolic (resp. hyperbolic) if it is the orthogonal sum of metabolic (resp. hyperbolic) planes. It is not difficult to see that a form $\beta$ is hyperbolic iff $D_{F}(\beta)=\{0\}$.

If the Gram matrix of a form $\beta$ with respect to a certain basis is a diagonal matrix with entries $a_{i}, 1 \leq i \leq n=\operatorname{dim}(\beta)$, then we write $\beta \cong\left\langle a_{1}, \cdots, a_{n}\right\rangle$. Such a diagonalization exists iff $\beta$ is not hyperbolic, by virtue of the relations

$$
\left(\begin{array}{ll}
a & 1 \\
1 & 0
\end{array}\right) \cong\langle a, a\rangle \text { and }\langle a\rangle \perp \mathbb{H} \cong\langle a, a, a\rangle \text {. }
$$


A form $\beta$ can be decomposed as $\beta \cong \beta_{\text {an }} \perp \beta_{m}$ with $\beta_{\text {an }}$ anisotropic and $\beta_{m}$ metabolic. $\beta_{\mathrm{an}}$ is uniquely determined up to isometry, but generally not $\beta_{m}$, as follows easily from the above relations.

Two forms $\beta$ and $\beta^{\prime}$ are called Witt equivalent if there are metabolic spaces $M$ and $M^{\prime}$ such that $\beta \perp M \cong \beta^{\prime} \perp M^{\prime}$. The equivalence classes together with addition induced by $\perp$ and multiplication induced by $\otimes$ define the Witt ring of $F$ denoted by $W F$. By abuse of notation, if $\beta$ is a form over $F$, we will also denote its class in $W F$ by $\beta$. Every class can be represented by an anisotropic form which is uniquely determined up to isometry.

An $n$-fold bilinear Pfister form (or $n$-Pfister for short) is a form of type

$$
\left\langle\left\langle a_{1}, \cdots, a_{n}\right\rangle\right\rangle:=\left\langle 1, a_{1}\right\rangle \otimes \cdots \otimes\left\langle 1, a_{n}\right\rangle
$$

for some $a_{i} \in F^{*}$. A bilinear Pfister form $\pi$ is round, i.e., $\pi \cong x \pi$ for all $x \in D_{F}(\pi)^{*}$ (see [2, IV.2.8]), and as an easy consequence one has that $\pi$ is isotropic iff $\pi$ is metabolic.

If $\beta$ is a form over $F$ and if $K / F$ is a field extension, then we write $\beta_{K}$ for the form obtained by scalar extension. This induces a natural ring homomorphism $W F \rightarrow W K$ whose kernel will be denoted by $W(K / F)$. In analogy to the definition of excellence of field extensions in the theory of quadratic forms in characteristic $\neq 2$ as defined in $[3$, we say that $K / F$ is excellent if for any form $\beta$ over $F$ there exists a form $\gamma$ over $F$ with $\left(\beta_{K}\right)_{\text {an }} \cong \gamma$, in other words, the anisotropic part of $\beta$ over $K$ is defined over $F$.

The annihilator ideal of a form $\beta$ over $F$ is given by

$$
\operatorname{ann}_{F}(\beta)=\{\psi \in W F \mid \beta \otimes \psi=0 \in W F\} .
$$

\section{WitT KERNELS AND EXCELLENCE FOR EXTENSIONS OF EXPONENT ONE}

Let us now turn to the proof of the main theorem. Throughout this section, $K / F$ will be a field extension with $K^{2} \subset F$. We define

$$
J_{K / F}=\text { the ideal in } W F \text { generated by the forms }\langle 1, t\rangle, t \in K^{* 2} \text {. }
$$

Lemma 3.1. $J_{K / F} \subset W(K / F)$.

Proof. If $t \in K^{* 2}$, then clearly $\langle 1, t\rangle_{K} \cong\langle 1,1\rangle_{K}$ is metabolic and hence $\langle 1, t\rangle_{K}=$ $0 \in W K$.

Proposition 3.2. Let $\varphi$ be an anisotropic form over $F$. Then $\varphi_{K}$ is isotropic iff there exist forms $\mu \in J_{K / F}$ and $\psi$ over $F$ with $\operatorname{dim} \psi<\operatorname{dim} \varphi$ such that

$$
\varphi=\psi+\mu \in W F .
$$

Proof. If $\varphi=\psi+\mu \in W F$ as in the statement of the proposition, then $\varphi_{K}=\psi_{K} \in$ $W K$ by the previous lemma, hence $\left(\varphi_{K}\right)_{\text {an }} \cong\left(\psi_{K}\right)_{\text {an }}$, implying the isotropy of $\varphi_{K}$ by comparing dimensions.

Conversely, let $\varphi$ be an anisotropic form over $F$ with $\varphi_{K}$ isotropic. Then we may assume that $\varphi \cong\left\langle a_{0}, \cdots, a_{n}\right\rangle$ with $a_{i} \in F^{*}$ and $n \geq 1$. For $\emptyset \neq I \subset\{0, \cdots, n\}$, let

$$
\varphi_{I}:=\frac{1}{i \in I}\left\langle a_{i}\right\rangle .
$$

We may assume that the form $\varphi_{I}$ is anisotropic over $K$ for any $I \neq\{0, \cdots, n\}$, for otherwise it would clearly suffice to prove the statement in the proposition with $\varphi$ replaced by $\varphi_{I}$ which is of smaller dimension. Let us call this assumption (X). 
Now the isotropy of $\varphi_{K}$ implies that there exist $t_{i}=x_{i}^{2} \in K^{2} \subset F, 0 \leq i \leq n$, not all $t_{i}$ equal to 0 , such that

$$
\sum_{i=0}^{n} a_{i} t_{i}=0
$$

and by assumption (X), we have $t_{i} \neq 0$ for $0 \leq i \leq n$. In particular, after dividing by $t_{0}$, we may assume that $t_{0}=1$. By putting $A_{0}=0$ and $A_{k}=\sum_{i=k}^{n} a_{i} t_{i}$ for $1 \leq k \leq n$, we have $a_{0}=A_{1}$ by equation (3.1), and it follows from assumption (X) that

$$
A_{k}=a_{k-1} t_{k-1}+A_{k-1} \neq 0 \text { for } 1 \leq k \leq n .
$$

Now if $u, v \in F^{*}$ such that $u+v \in F^{*}$ (i.e. $u \neq v$ as $\operatorname{char}(F)=2$ ), then one verifies easily that $\langle u, v\rangle \cong\langle u+v,(u+v) u v\rangle$, or

$$
\langle u+v\rangle=\langle u, v,(u+v) u v\rangle \in W F .
$$

Now define

$$
\alpha_{k}:=a_{k-1} t_{k-1} A_{k-1} A_{k}=\left(a_{k-1} t_{k-1}+A_{k}\right) a_{k-1} t_{k-1} A_{k} \quad \text { for } 2 \leq k \leq n .
$$

Equations (3.2) and (3.3) yield in WF:

$$
\begin{aligned}
\varphi & =\left\langle a_{0}, a_{1}, \cdots, a_{n}\right\rangle \\
& =\left\langle A_{1}, a_{1}, \cdots, a_{n}\right\rangle \\
& =\left\langle a_{1} t_{1}+A_{2}, a_{1}, a_{2}, \cdots, a_{n}\right\rangle \\
& =\left\langle a_{1} t_{1}, A_{2}, \alpha_{2}, a_{1}, a_{2}, \cdots, a_{n}\right\rangle \\
& =a_{1}\left\langle 1, t_{1}\right\rangle+\left\langle A_{2}, a_{2}, \cdots, a_{n}\right\rangle+\left\langle\alpha_{2}\right\rangle .
\end{aligned}
$$

Repeating this for $\left\langle A_{2}, a_{2}, \cdots, a_{n}\right\rangle$ and so on, and using in the last step that $\left\langle A_{n}, a_{n}\right\rangle \cong a_{n}\left\langle 1, t_{n}\right\rangle$, we get

$$
\begin{aligned}
\varphi & =a_{1}\left\langle 1, t_{1}\right\rangle+a_{2}\left\langle 1, t_{2}\right\rangle+\left\langle A_{3}, a_{3}, \cdots, a_{n}\right\rangle+\left\langle\alpha_{2}, \alpha_{3}\right\rangle \\
& \vdots \\
& =\sum_{i=1}^{n} a_{i}\left\langle 1, t_{i}\right\rangle+\left\langle\alpha_{2}, \cdots, \alpha_{n}\right\rangle .
\end{aligned}
$$

The proof is now finished by putting $\psi:=\left\langle\alpha_{2}, \cdots, \alpha_{n}\right\rangle$ and $\mu=\perp_{i=1}^{n} a_{i}\left\langle 1, t_{i}\right\rangle$.

Corollary 3.3. Let $\varphi$ be a form over $F$. Then there exist forms $\psi$ and $\mu$ over $F$ with $\psi_{K}$ anisotropic (possibly $\operatorname{dim} \psi=0$ ) and $\mu \in J_{K / F}$, such that $\varphi=\psi+\mu \in W F$. In particular, $\left(\varphi_{K}\right)_{\mathrm{an}} \cong \psi_{K}$.

Proof. If $\varphi_{K}$ is anisotropic, then we can put $\psi:=\varphi$ and $\mu=0$.

If $\varphi_{K}$ is isotropic, then we apply the above proposition to find forms $\mu^{\prime} \in J_{K / F}$ and $\psi^{\prime}$ over $F$ with $\operatorname{dim} \psi^{\prime}<\operatorname{dim} \varphi$ such that $\varphi=\psi^{\prime}+\mu^{\prime} \in W F$. If $\psi_{K}^{\prime}$ is anisotropic, we are done, otherwise we conclude by induction on the dimension applied to $\operatorname{dim} \psi^{\prime}$.

Now by Lemma 3.1 we have $\mu_{K}=0$, hence $\varphi_{K}=\psi_{K} \in W K$. The anisotropy of $\psi_{K}$ yields $\left(\varphi_{K}\right)_{\text {an }} \cong \psi_{K}$.

Our main theorem from the Introduction and the excellence of $K / F$ are now immediate consequences of the preceding corollary and Lemma 3.1

Theorem 3.4. (i) $W(K / F)=J_{K / F}$.

(ii) $K / F$ is excellent. 


\section{Annihilator ideals for Pfister forms}

Consider an $n$-Pfister

$$
\pi=\left\langle\left\langle a_{1}, \cdots, a_{n}\right\rangle\right\rangle=\underset{\emptyset \subset I \subset\{1, \cdots, n\}}{\perp}\left\langle a_{I}\right\rangle,
$$

where $a_{I}=\prod_{i \in I} a_{i}$. We associate to this Pfister form the field $K=F\left(\sqrt{a_{1}}, \cdots, \sqrt{a_{n}}\right)$ which is purely inseparable of exponent 1 , and we have $K^{2}=F^{2}\left(a_{1}, \cdots, a_{n}\right)$. It is clear that

$$
\sum_{\emptyset \subset I \subset\{1, \cdots, n\}} a_{I} F^{2}=D_{F}(\pi)_{0}=K^{2} .
$$

We call $K$ the root field of $\pi$ (as it consists exactly of all square roots of elements in $\left.D_{F}(\pi)_{0}\right)$. Note that $\pi$ is anisotropic iff the $a_{I}$ are $F^{2}$-linearly independent, which in turn is equivalent to saying that $[K: F]=\left[K^{2}: F^{2}\right]=2^{n}$.

Conversely, if $K / F$ is a purely inseparable extension of exponent 1 and degree $2^{n}$, then we can find $a_{1}, \cdots, a_{n} \in F^{*}$ such that $K=F\left(\sqrt{a_{1}}, \cdots, \sqrt{a_{n}}\right)$. We then call $\pi=\left\langle\left\langle a_{1}, \cdots, a_{n}\right\rangle\right\rangle$ a Pfister form associated to $K / F$, and we have $D_{F}(\pi)_{0}=$ $K^{2}$. Note that this Pfister form is generally not unique up to isometry. For if we choose different elements $b_{1}, \cdots, b_{n} \in F$ with $K=F\left(\sqrt{b_{1}}, \cdots, \sqrt{b_{n}}\right)$, then generally $\left\langle\left\langle b_{1}, \cdots, b_{n}\right\rangle\right\rangle \not\left\langle\left\langle a_{1}, \cdots, a_{n}\right\rangle\right\rangle$.

For example, if $F=\mathbb{F}_{2}(T)$ is the rational function field in one variable $T$ over the field with two elements, and if $K=F(\sqrt{T})=F(\sqrt{1+T})$, then $\langle\langle T\rangle\rceil \not\langle 1+T\rangle\rangle$ by comparing determinants.

We will focus on annihilator ideals for Pfister forms.

For the remainder of this section, $\pi$ will be an anisotropic $n$-Pfister, and $K$ will be the root field of $\pi$.

Lemma 4.1. $J_{K / F} \subset \operatorname{ann}_{F}(\pi)$.

Proof. $J_{K / F}$ is generated by elements of type $\langle 1, t\rangle$ with $t \in K^{* 2}=D_{F}(\pi)^{*}$. Since $\pi$ is round, we have $t \pi \cong \pi$, i.e. $\langle 1, t\rangle \otimes \pi=0 \in W F$ for all $t \in D_{F}(\pi)^{*}$. This clearly implies $J_{K / F} \subset \operatorname{ann}_{F}(\pi)$.

Proposition 4.2. Let $\varphi=\left\langle a_{0}, \cdots, a_{n}\right\rangle$ be an anisotropic form over $F$. Then $\pi \otimes \varphi$ is isotropic iff $\varphi_{K}$ is isotropic.

Proof. $\pi \otimes \varphi \cong \perp_{i=0}^{n} a_{i} \pi$ is isotropic iff there exist $t_{i} \in D_{F}(\pi)_{0}=K^{2}, 0 \leq i \leq n$, not all $t_{i}$ equal to 0 (here, we use the anisotropy of $\pi$ ), such that $\sum_{i=0}^{n} a_{i} t_{i}=0$. Since $t_{i}=x_{i}^{2}$ for a uniquely determined $x_{i} \in K$, this immediately shows that the isotropy of $\pi \otimes \varphi$ is equivalent to the isotropy of $\varphi_{K}$.

Corollary 4.3. Let $\varphi$ be a form over $F$. Then there exist forms $\psi$ and $\mu$ over $F$ with $\pi \otimes \psi$ anisotropic (possibly $\operatorname{dim} \psi=0$ ) and $\mu \in J_{K / F}$, such that $\varphi=\psi+\mu \in W F$. In particular, $(\pi \otimes \varphi)_{\text {an }} \cong \pi \otimes \psi$.

Proof. By Corollary 3.3 there exist forms $\psi$ and $\mu$ over $F$ with $\psi_{K}$ anisotropic (possibly $\operatorname{dim} \psi=0$ ) and $\mu \in J_{K / F}$, such that $\varphi=\psi+\mu \in W F$. Now $\pi \otimes \mu=$ $0 \in W F$ by Lemma 4.1, and $\pi \otimes \psi$ is anisotropic by Proposition 4.2. Hence $\pi \otimes \varphi=\pi \otimes \psi \in W F$ and thus $(\pi \otimes \varphi)_{\text {an }} \cong \pi \otimes \psi$.

This corollary together with Lemma4.1 and Theorem 3.4(i) immediately implies

Theorem 4.4. $\operatorname{ann}_{F}(\pi)=J_{K / F}=W(K / F)$. 
Corollary 4.5. Let $E / F$ be purely inseparable of exponent 1 . For any finite subextension $L$ of $F$ inside $E$, let $\pi^{(L)}$ be the Pfister form associated to $L / F$. Then

$$
W(E / F)=\bigcup_{\substack{F \subset L \subset E \\ L / F \text { finite }}} \operatorname{ann}_{F}\left(\pi^{(L)}\right) .
$$

Proof. Every form $\varphi$ over $F$ that becomes metabolic over $E$ will already become so over some finite subextension of $E / F$. Hence, $W(E / F)=\bigcup_{L} W(L / F)$, where $L$ ranges over all finite subextensions of $F$ inside $E$. The claim now follows by applying Theorem 4.4

\section{A generalization}

Our results on isotropy over purely inseparable (finite) extensions can be extended to a wider class of algebraic extensions, not necessarily purely inseparable. We will often refer to [4, where diagonal forms of degree $p$ over fields of characteristic $p>0$ are studied. In our situation, the characteristic is 2 , and diagonal forms of degree 2 are then nothing else but totally singular quadratic forms. The results in 4 can now be applied by noting that the isotropy of a bilinear form $\beta$ is by definition the same as the isotropy of the associated totally singular quadratic form $q$ given by $q(x)=\beta(x, x)$.

The case of separable extensions can be disposed of immediately.

Lemma 5.1. Let $\varphi$ be an anisotropic form over $F$ and let $K$ be a separable extension of $F$. Then $\varphi_{K}$ is anisotropic.

Proof. [4, Prop. 5.3].

This result applies to separable extensions in the widest sense, namely extensions $K / F$ which can be written as a purely transcendental extension (of arbitrary transcendence degree) followed by a separable algebraic extension (not necessarily finite).

Now let $K / F$ be an algebraic extension. We still assume $\operatorname{char}(F)=2$, although all the field-theoretic definitions and results in the next few paragraphs make sense in arbitrary (positive) characteristic.

It is well known that $K / F$ can be written as $K / L / F$ with $L / F$ separable and $K / L$ purely inseparable. If it can also be written as $K / M / F$ with $M / F$ purely inseparable and $K / M$ separable; then the field extension $K / F$ is said to be balanced. Now generally, finite extensions need not be balanced. But if $K / F$ is balanced, then the $M$ above is necessarily the maximal purely inseparable extension of $F$ in $K$.

More precisely, if $L$ (resp. $M$ ) is the maximal separable (resp. purely inseparable) extension of $F$ inside $K$, then $K / F$ is balanced iff $K / M$ is separable iff $K=L M$. For example, every finite normal extension $K / F$ is balanced (see, e.g., [5, Th. 46]).

Recall that elements $b_{1}, \cdots, b_{m} \in F$ are called 2-independent if $\left[F^{2}\left(b_{1}, \cdots, b_{m}\right)\right.$ : $\left.F^{2}\right]=2^{m}$, or, equivalently $\left[F\left(\sqrt{b_{1}}, \cdots, \sqrt{b_{m}}\right): F\right]=2^{m}$.

Now suppose that $K / F$ is a finite purely inseparable extension of degree $2^{r}>1$. Then there exist elements $a_{1}, \cdots, a_{n} \in F, \alpha_{1}, \cdots, \alpha_{n} \in K$ and integers $r_{1}, \cdots, r_{n} \geq$ 1 such that $a_{i}=\alpha_{i}^{2^{r_{i}}}$ and $K=F\left(\alpha_{1}, \cdots, \alpha_{n}\right)$. One can show that

$$
2^{r}=[K: F]=2^{r_{1}+\cdots+r_{n}}
$$


if and only if $\left[F\left(\sqrt{a_{1}}, \cdots, \sqrt{a_{n}}\right): F\right]=2^{n}$, i.e., the $a_{i}$ are 2-independent (see 4, Prop. 5.7]). If we are in this situation, then we have

$$
K=\prod_{i=1}^{n} F\left(\alpha_{i}\right) \cong F\left(\alpha_{1}\right) \otimes_{F} \cdots \otimes_{F} F\left(\alpha_{n}\right),
$$

and we say that $K / F$ admits a higher 2-basis $\left\{\alpha_{1}, \cdots, \alpha_{n}\right\}$. Generally, finite purely inseparable extensions do not admit higher 2-bases (see [9]).

We now have all the ingredients to generalize the results from the previous sections to a much wider class of field extensions.

Theorem 5.2. Let $K / F$ be a finite balanced extension and let $M$ be the maximal purely inseparable extension of $F$ inside $K$. Suppose that $M / F$ admits a higher 2basis $\left\{\alpha_{1}, \cdots, \alpha_{n}\right\} \subset M$, and let $\left\{a_{1}, \cdots, a_{n}\right\} \subset F$ be 2-independent with $a_{i}=\alpha_{i}^{2^{r_{i}}}$ with integers $r_{1}, \cdots, r_{n} \geq 1$. Let $L=F\left(\sqrt{a_{1}}, \cdots, \sqrt{a_{n}}\right)$.

Then $\pi=\left\langle\left\langle a_{1}, \cdots, a_{n}\right\rangle\right\rangle$ is anisotropic, and for an anisotropic form $\varphi$ over $F$, the following are equivalent:

(i) $\varphi_{K}$ is isotropic;

(ii) $\varphi_{M}$ is isotropic;

(iii) $\varphi_{L}$ is isotropic;

(iv) $\pi \otimes \varphi$ is isotropic.

In particular, $K / F$ is excellent and $W(K / F)=W(M / F)=W(L / F)=\operatorname{ann}_{F}(\pi)$.

Proof. The anisotropy of $\pi$ is clear (see the introductory remarks in Section 4).

The equivalence of (i) and (ii) follows from the assumption that $K / F$ is balanced, which in turn means that $K / M$ is separable, implying that a form (defined over $M)$ is anisotropic over $K$ iff it is so over $M$ by Lemma 5.1 .

The equivalence of (ii) and (iii) has been proved in [4, Th. 5.9], and Proposition 4.2 yields the equivalence of (iii) and (iv).

The excellence follows now readily from Corollary 3.3. The equality of the Witt kernels is also immediate, and the proof is finished by invoking Theorem 4.4.

We conclude by giving an example of a finite algebraic extension $K / F$ which is not excellent.

Example 5.3. Let $F=\mathbb{F}_{2}(a, b, c)$ be the rational function field in the variables $a, b, c$ over $\mathbb{F}_{2}$. One readily checks that $X^{3}+a X^{2}+b X+c \in F[X]$ is irreducible over $F$. Hence,

$$
P(X)=X^{6}+a X^{4}+b X^{2}+c \in F[X]
$$

is irreducible over $F$. Let $\alpha$ be a root of $P(X)$ in some algebraic closure of $F$, and put $K=F(\alpha)$. We have $[K: F]=6$ and clearly $K / F$ is neither separable nor purely inseparable.

Now let

$$
\varphi=\langle 1, a, b, c\rangle \quad \text { and } \quad \pi=\langle\langle a, b, c\rangle\rangle \text {. }
$$

One easily sees that $\varphi$ and $\pi$ are anisotropic as $a, b, c$ are 2-independent. Furthermore, $\varphi_{K}$ is isotropic because we have

$$
\left(\alpha^{3}\right)^{2}+a\left(\alpha^{2}\right)^{2}+b \alpha^{2}+c .1^{2}=0 .
$$

Suppose that there exists a form $\psi$ over $F$ with $\left(\varphi_{K}\right)_{\text {an }} \cong \psi_{K}$. Then $\operatorname{dim} \psi \in\{0,2\}$. Let

$$
\eta \cong(\varphi \perp \psi)_{\mathrm{an}} .
$$


By comparing dimensions, we have $2 \leq \operatorname{dim} \eta \leq 6$. Furthermore, by construction, $\eta_{K}$ is metabolic. Let $\widehat{\eta}$ (resp. $\widehat{\pi}$ ) be the anisotropic totally singular quadratic form given by $\widehat{\eta}(x)=\eta(x, x)$ (resp. $\widehat{\pi}(x)=\pi(x, x))$. Since $\eta_{K}$ is metabolic, we have that $\widehat{\eta}_{K}$ is 2-split in the terminology of [4, Def. 2.11].

Now the coefficients of $P(X)$ are $1, a, b, c$, and we have $\left[F^{2}(a, b, c): F^{2}\right]=8$ and $K \cong F[X] / P(X)$. We then can invoke [4, Th. 6.10] to get that $\widehat{\eta} \cong \widehat{\pi} \otimes \widehat{\gamma}$ for some totally singular quadratic form $\widehat{\gamma}$. In particular, $\operatorname{dim} \eta=\operatorname{dim} \widehat{\eta}$ is divisible by $\operatorname{dim} \pi=\operatorname{dim} \widehat{\pi}=8$, a contradiction. Thus, $\left(\varphi_{K}\right)_{\text {an }}$ is not defined over $F$.

\section{REFERENCES}

1. R. Baeza, Ein Teilformensatz für quadratische Formen in Charakteristik 2, Math. Z. 135 (1974), 175-184. MR0337765 (49:2534)

2. R. Baeza, Quadratic forms over semi-local rings, Lecture Notes in Mathematics, vol. 655, Springer-Verlag, 1976. MR0491773 (58:10972)

3. R. Elman, T.Y. Lam, and A. Wadsworth, Amenable fields and Pfister extensions, Proc. of Quadratic Forms Conference, Queen's Univ., Kingston, Ont., 1976 (G. Orzech, ed.). Queen's Papers in Pure and Applied Mathematics No. 46 (1977), 445-491. MR0560497 (58:27756)

4. D. Hoffmann, Diagonal forms of degree $p$ in characteristic $p$, Algebraic and Arithmetic Theory of Quadratic Forms, 135-183, Contemp. Math., 344, Amer. Math. Soc., Providence, RI, 2004. MR2058673

5. I. Kaplansky, Fields and Rings, 2nd ed., Chicago Lectures in Mathematics, The University of Chicago Press, Chicago, Ill.-London, 1972. MR0349646 (50:2139)

6. K. Kato, Symmetric bilinear forms, quadratic forms and Milnor K-theory in characteristic two, Invent. Math. 66 (1982), 493-510. MR0662605 (83i:10027)

7. A. Laghribi, Witt kernels of quadratic forms for multiquadratic extensions in characteristic 2 , preprint 2004.

8. A. Pfister, Quadratic Forms with Applications to Algebraic Geometry and Topology, London Math. Soc. Lecture Note Series, vol. 217, Cambridge University Press, Cambridge, 1995. MR1366652 (97c:11046)

9. M. Weisfeld, Purely inseparable extensions and higher derivations, Trans. Amer. Math. Soc. 116 (1965), 435-449. MR0191895 (33:122)

School of Mathematical Sciences, University of Nottingham, University Park, NotTingham NG7 2RD, United Kingdom

E-mail address: detlev.hoffmann@nottingham.ac.uk 